\title{
Borel classes of uniformizations of sets with large sections
}

by

\author{
Petr Holický (Praha)
}

\begin{abstract}
We give several refinements of known theorems on Borel uniformizations of sets with "large sections". In particular, we show that a set $B \subset[0,1] \times[0,1]$ which belongs to $\boldsymbol{\Sigma}_{\alpha}^{0}, \alpha \geq 2$, and which has all "vertical" sections of positive Lebesgue measure, has a $\boldsymbol{\Pi}_{\alpha}^{0}$ uniformization which is the graph of a $\boldsymbol{\Sigma}_{\alpha}^{0}$-measurable mapping. We get a similar result for sets with nonmeager sections. As a corollary we derive an improvement of Srivastava's theorem on uniformizations for Borel sets with $G_{\delta}$ sections.
\end{abstract}

1. Introduction. We are going to answer a question posed by Piotr Borodulin-Nadzieja, namely what can be said about the Borel class of Borel measurable selections of Borel sets with sections of positive Lebesgue measure. The existence of a Borel uniformization in such a case was shown by Blackwell and Ryll-Nardzewski [1]. The existence of Borel uniformizations of sets with large sections in the sense of category was proved by Sarbadhikari [10]. An abstract version can be found in [4, Theorem 18.6]. Mauldin [8] proved the existence of Borel parametrizations in the above cases. In fact, all the above mentioned results deal with rather more general situations. We will also deal with a slightly more general setting.

We get uniformization theorems for Borel sets $B \subset X \times Y$, for $X$ and $Y$ Polish, with sections $B_{x}=\{y \in Y:(x, y) \in B\}$ of positive probability $\mu\left(x, B_{x}\right)$ with respect to suitable probability kernels $\mu$ and for Borel sets with sections $B_{x}$ nonmeager (i.e., not of the first category) in suitable Baire supersets $F(x) \subset Y$. In both cases we get some information about the Borel class of the corresponding selection. We also get a continuum of such pairwise disjoint uniformizations with their union parametrized by $X \times\{0,1\}^{\mathbb{N}}$ in a Borel isomorphic way. As a corollary of the "category case", we get a modification of the Srivastava selection theorem for $G_{\delta}$-valued mappings (see [12, Theorem 4.1]). Thus we get information about the Borel class of the selection also in this case. Our proof is inspired by that of Kechris (see

2010 Mathematics Subject Classification: Primary 54H05; Secondary 54C65, 54E50.

Key words and phrases: Borel classes, sets with large sections, uniformizations, selections. 
[4, Exercise 18.20(iv) and the hint to it] or [11, Theorem 5.9.2]). We also get a version of this theorem for selectors of partitions.

The main methods we need to get our refinements are well-known. The first involves a finer description of Borel sets of a given class using a particular scheme of subsets (cf. [4, Theorem 22.21], or [7, Theorem 2.2] for the nonseparable case). Another one is the method of getting a selection used in [6] which we only slightly modify. As is well-known, the latter result yields some information about the class of the selection, so it is no surprise that we get our results in this way (cf. also [9] in this context).

Since the methods work almost without any further effort also for $X$ nonseparable, we formulate our results in this more general setting.

2. Projections along large sections. We modify the elegant proofs of the preservation of Borelness when projecting sets with large sections to get some information about the Borel class of the projection. In the case of large sections in the sense of category we improve a result of Montgomery (see [4, Exercise 22.22]), as a revision of the proof of the theorem by Montgomery and Novikov (see [4, Theorem 16.1]). Similarly, we also get the desired modification of the property "Borel on Borel" from [4, Definition 18.5] for the case of Borel probability kernels.

We use the standard notation $\mathcal{B}(Y)$ for the set of all Borel subsets of the topological space $Y$. We also denote by $\boldsymbol{\Sigma}_{\alpha}^{0}, \boldsymbol{\Pi}_{\alpha}^{0}, \boldsymbol{\Delta}_{\alpha}^{0}$ the additive, multiplicative, and ambiguous classes for $\alpha \geq 1$. We write, e.g., $\boldsymbol{\Sigma}_{\alpha}^{0}(Y)$ for the family of all Borel sets of class $\boldsymbol{\Sigma}_{\alpha}^{0}$ in $Y$. For this notation, see e.g. [4.

A family $\mathcal{D}$ of sets in a metric space $X$ is discrete if every $x \in X$ has a neighborhood intersecting at most one element of $\mathcal{D}$. The family is $\sigma$ discrete if it is the union of countably many discrete families. It is an easy, and well-known, observation that $\mathcal{D}_{1} \wedge \cdots \wedge \mathcal{D}_{k}:=\left\{D_{1} \cap \cdots \cap D_{k}: D_{j} \in\right.$ $\left.\mathcal{D}_{j}, j=1, \ldots, k\right\}$ is $(\sigma-)$ discrete if every $\mathcal{D}_{j}$ is $(\sigma-)$ discrete. We repeatedly use the fact that the union of a discrete family of sets in $\boldsymbol{\Sigma}_{\alpha}^{0}(X)$, for a metric space $X$, is in $\boldsymbol{\Sigma}_{\alpha}^{0}$ for every countable ordinal $\alpha \geq 1$ (see [5, $\S 30, \mathrm{X}$, Theorems 3 and 4]).

To point out what assumptions are needed, we formulate the following lemma in a more general setting than we need below. Recall that a Borel measure $\mu$ on a topological space $Y$ is $\tau$-additive if $\sup \{\mu(A): A \in \mathcal{A}\}=$ $\mu(\bigcup \mathcal{A})$ for every family of open sets in $Y$ which is upwards directed (cf. [2, Definition 2.3]). Every Radon measure is $\tau$-additive ([2, Proposition 6.9]) and every finite Borel measure on a Polish space is Radon ([4, Theorem 17.11]).

Lemma 2.1. Let $X$ be a metrizable space, $Y$ be a topological space, and $\mu: X \times \mathcal{B}(Y) \rightarrow[0,1]$ be such that 
(a) $\mu(x, \cdot)$ is a Borel $\tau$-additive probability on $Y$ for every $x \in X$,

(b) $\{x \in X: \mu(x, H)>r\}$ is $\Sigma_{\alpha_{0}}^{0}$-measurable in $X\left(1 \leq \alpha_{0}<\omega_{1}\right)$ for every open $H \subset Y$ and $r \in \mathbb{R}$.

Let $B \subset X \times Y$ be in $\boldsymbol{\Sigma}_{\alpha}^{0}(X \times Y)$ for some $\alpha$ with $1 \leq \alpha<\omega_{1}$. Then the set $\left\{x \in X: \mu\left(x, B_{x}\right)>0\right\}$ is in $\boldsymbol{\Sigma}_{\alpha^{*}}^{0}(X)$, where $\alpha^{*}=\alpha_{0}+\alpha$ if $\alpha \geq \omega$ and $\alpha^{*}=\alpha_{0}+(\alpha-1)$ if $\alpha<\omega$.

Proof. We shall show that $\pi_{\mu}^{*}(B, r):=\left\{x \in X: \mu\left(x, B_{x}\right)>r\right\}$ is in $\Sigma_{\alpha^{*}}^{0}(X)$ for every $r \in \mathbb{R}$ by induction on $\alpha$ (with $\alpha_{0}$ fixed).

Let $B$ be an open subset of $X \times Y$. There is a $\sigma$-discrete base $\mathcal{U}$ of open subsets of $X$ (see [5, $\S 21$, XVI, Corollary 1a]). So $B=\bigcup\left\{U_{a} \times W_{a}: a \in I\right\}$, where each $W_{a}$ is open in $Y$, and the family $\left\{U_{a}\right\}_{a \in I} \subset \mathcal{U}$ is $\sigma$-discrete in $X$. We use the $\tau$-additivity of $\mu(x, \cdot)$ for each $x \in X$. We apply it to the upwards directed family of open sets $\mathcal{A}_{x}=\left\{W_{a_{1}} \cup \cdots \cup W_{a_{k}}: x \in\right.$ $\left.U_{a_{1}} \cap \cdots \cap U_{a_{k}}, a_{1}, \ldots, a_{k} \in I, k \in \mathbb{N}\right\}$. Since $B_{x}=\bigcup \mathcal{A}_{x}$ by the equality $B=\bigcup\left\{U_{a} \times W_{a}: a \in I\right\}$, we have $\mu\left(x, B_{x}\right)=\sup \left\{\mu(x, A): A \in \mathcal{A}_{x}\right\}$. Therefore $\mu\left(x, B_{x}\right)>r$ if and only if there are $a_{1}, \ldots, a_{k} \in I$ such that $x \in U_{a_{1}} \cap \cdots \cap U_{a_{k}}$ and $\mu\left(x, W_{a_{1}} \cup \cdots \cup W_{a_{k}}\right)>r$. Put $U\left(a_{1}, \ldots, a_{k}, r\right)=$ $\left\{x \in U_{a_{1}} \cap \cdots \cap U_{a_{k}}: \mu\left(x, W_{a_{1}} \cup \cdots \cup W_{a_{k}}\right)>r\right\}$. The family $\left\{U\left(a_{1}, \ldots, a_{k}, r\right)\right.$ : $\left.k \in \mathbb{N}, a_{1}, \ldots, a_{k} \in I\right\}$ forms a $\sigma$-discrete cover of the set $\pi_{\mu}^{*}(B, r)$ by sets from $\Sigma_{\alpha_{0}}^{0}(X)$ (this easy fact follows from the remark before the lemma). Thus $\pi_{\mu}^{*}(B, r)$ is in $\boldsymbol{\Sigma}_{\alpha_{0}}^{0}(X)$, which is our claim for $\alpha=1$.

Let $\alpha>1$ and suppose the claim is valid for all Borel sets $C$ of additive class $\beta$ for every $1 \leq \beta<\alpha$. Let $B \subset X \times Y$ be in $\Sigma_{\alpha}^{0}(X \times Y)$. Thus there are $B_{n} \in \Pi_{\beta_{n}}^{0}(X \times Y)$ with $B_{n} \subset B_{n+1}$ and $1 \leq \beta_{n}<\alpha$ such that $B=\bigcup_{n \in \mathbb{N}} B_{n}$. Now

$$
\begin{aligned}
\pi_{\mu}^{*}(B, r) & =\bigcup_{n=1}^{\infty} \pi_{\mu}^{*}\left(B_{n}, r\right)=\bigcup_{n=1}^{\infty}\left\{x \in X: \mu\left(x,\left(B_{n}^{c}\right)_{x}\right)<1-r\right\} \\
& =\bigcup_{n=1}^{\infty} \bigcup_{p=1}^{\infty}\left\{x \in X: \mu\left(x,\left(B_{n}^{c}\right)_{x}\right) \leq 1-r-1 / p\right\} \\
& =\bigcup_{n=1}^{\infty} \bigcup_{p=1}^{\infty} X \backslash\left\{x \in X: \mu\left(x,\left(B_{n}^{c}\right)_{x}\right)>1-r-1 / p\right\} .
\end{aligned}
$$

By the induction hypothesis, $\left\{x \in X: \mu\left(x,\left(B_{n}^{c}\right)_{x}\right)>1-r-1 / p\right\} \in \mathbf{\Sigma}_{\beta_{n}^{*}}^{0}(X)$. It follows that $\pi_{\mu}^{*}(B, r)$ is a countable union of sets in $\boldsymbol{\Pi}_{\beta_{n}^{*}}^{0}(X) \subset \boldsymbol{\Sigma}_{\alpha^{*}}^{0}(X)$.

We use the notion of Baire space in the sense of [4, Definition 8.2], i.e., it is a topological space with no meager nonempty open subset. Thus the empty space is a Baire space and let us consider $\emptyset$ to be meager even in the empty space for formal reasons. 
The symbol $\mathcal{P}(Y)$ denotes the power set of $Y$. The multivalued mapping $F:(X, \mathcal{A}) \rightarrow \mathcal{P}(Y)$, where $Y$ is a topological space, is lower $\mathcal{A}$-measurable whenever $F^{-1}(H):=\{x \in X: F(x) \cap H \neq \emptyset\} \in \mathcal{A}$ for every open $H \subset Y$. Here $\mathcal{A}$ might be a family of subsets of $X$ or a family of subsets of some superspace of $X$ etc. Moreover, $F$ is upper $\mathcal{A}$-measurable if $F_{-1}(H):=\{x \in$ $X: F(x) \subset H\} \in \mathcal{A}$ for every open $H \subset Y$. Similarly, we say that $f$ : $X \rightarrow Y$ is $\mathcal{A}$-measurable if $f^{-1}(H) \in \mathcal{A}$ for every open subset $H$ of $Y$ $\left(H \in \boldsymbol{\Sigma}_{1}^{0}(Y)\right)$. The set graph $F:=\{(x, y) \in X \times Y: y \in F(x)\}$ is the graph of the multivalued mapping $F$.

Lemma 2.2. Let $X$ be a metrizable space, and $Y$ be a separable metrizable space. Let $F: X \rightarrow \mathcal{P}(Y)$ be lower $\Sigma_{\alpha_{0}}^{0}$-measurable $\left(1 \leq \alpha_{0}<\omega_{1}\right)$, with $F(x)$ a Baire subspace of $Y$ for every $x \in X$, and $B \subset$ graph $F$ of the type $\boldsymbol{\Sigma}_{\alpha}^{0}$ in $X \times Y, 1 \leq \alpha<\omega_{1}$. Then the set $\left\{x \in X: B_{x}\right.$ is not meager in $\left.F(x)\right\}$ is in $\boldsymbol{\Sigma}_{\alpha^{*}}^{0}$, where $\alpha^{*}$ is as in Lemma 2.1 .

The appearance of $F$ here is related to the modification of the proof of the Montgomery and Novikov Theorem suggested in the hint to [4, Exercise 18.20(iii)].

Proof. Let us first show the following claim.

Claim. $\pi_{F}^{*}(B, W):=\left\{x \in X: F(x) \cap B_{x} \cap W\right.$ is not meager in $\left.F(x) \cap W\right\}$ is in $\boldsymbol{\Sigma}_{\alpha^{*}}^{0}(X)$ if $B$ is in $\boldsymbol{\Sigma}_{\alpha}^{0}(X \times Y)$, for every open set $W \subset Y$.

We proceed by induction over $\alpha$. If $B=\bigcup\left\{U_{a} \times W_{a}: a \in I\right\}$, where $U_{a}$ and $W_{a}$ are open in $X$ and $Y$, respectively, and if $\left\{U_{a}\right\}_{a \in I}$ is $\sigma$-discrete, then $\pi_{F}^{*}(B, W)=\bigcup\left\{\left\{x \in U_{a}: F(x) \cap W_{a} \cap W \neq \emptyset\right\}: a \in I\right\}=\bigcup\left\{U_{a} \cap F^{-1}(W \cap\right.$ $\left.\left.W_{a}\right): a \in I\right\}$, which is in $\boldsymbol{\Sigma}_{\alpha_{0}}^{0}(X)$ by our assumptions. Here we have used the fact that no nonempty relatively open subset of $F(x)$ is meager in $F(x)$. Thus our claim for $\alpha=1$ is proved.

Let $\alpha>1$ and assume the claim to be valid for every $\beta \geq 1$ less than $\alpha$. Let $B \subset X \times Y$ be in $\boldsymbol{\Sigma}_{\alpha}^{0}(X \times Y)$. Thus there are $B_{n} \in \boldsymbol{\Pi}_{\beta_{n}}^{0}(X \times Y)$ with $\beta_{n}<\alpha$ such that $B=\bigcup_{n \in \mathbb{N}} B_{n}$. For a fixed open set $W \subset Y$ we get

$$
\begin{aligned}
& \pi_{F}^{*}(B, W)=\bigcup_{n \in \mathbb{N}} \pi_{F}^{*}\left(B_{n}, W\right) \\
&=\bigcup_{n \in \mathbb{N}} \bigcup_{\substack{W^{\prime} \in \mathcal{W} \\
W^{\prime} \subset W}}\left\{x \in X: F(x) \cap\left(B_{n}\right)_{x} \cap W^{\prime}\right. \\
&=\bigcup_{n \in \mathbb{N}} \bigcup_{\substack{W^{\prime} \in \mathcal{W} \\
W^{\prime} \subset W}}\left\{x \in X: F(x) \cap\left(B_{n}^{c}\right)_{x} \cap W^{\prime}\right. \\
&\text { is meager in } \left.F(x) \cap W^{\prime}\right\} \cap F^{-1}\left(W^{\prime}\right),
\end{aligned}
$$

where $\mathcal{W}$ is a countable base of $Y$ consisting of nonempty open sets. Here 
we have used the fact that a subset with the Baire property of a Baire space is nonmeager if and only if it is residual in some nonempty open subset (see, e.g., [4, Proposition 8.26]). Since the sets $F^{-1}\left(W^{\prime}\right)$ are in $\Sigma_{\alpha_{0}}^{0}(X)$ and the sets

$$
\pi_{F}^{*}\left(B_{n}^{c}, W^{\prime}\right)=X \backslash\left\{x \in X: F(x) \cap\left(B_{n}^{c}\right)_{x} \cap W^{\prime} \text { is meager in } F(x) \cap W^{\prime}\right\}
$$

are in $\boldsymbol{\Sigma}_{\beta_{n}^{*}}^{0}(X)$ by the induction assumption, $\pi_{F}^{*}(B, W)$ is in $\boldsymbol{\Sigma}_{\alpha^{*}}^{0}(X)$.

3. Uniformizations of sets with large sections. In the proof of the uniformization theorems below, we use the existence of a scheme of subsets of a Borel set in a complete metric space. Our requirements on it will be similar to but weaker than those for a Luzin scheme in [4, Theorem 22.21] because they will be sufficient for our purpose and the existence of such a scheme follows from published results even in the case of $\alpha=1$ for general Polish spaces $X$ and $Y$, as well as in the case of a nonseparable complete metric space $X$ and $\alpha \geq 1$. We recall some notation first.

If $\mathbb{D}$ is any set, $\mathbb{D}^{<\omega}$ denotes the set $\bigcup_{n=1}^{\infty} \mathbb{D}^{n} \cup\{\emptyset\}$ of finite sequences of elements of $\mathbb{D}$. For $s \in \mathbb{D}^{<\omega}$, the symbol $|s|$ stands for the length of $s$. We also write $s^{\prime} \succ s$ if $s^{\prime}$ is a strict extension of $s^{\prime}$ (i.e., if $s^{\prime}$ extends $s$ and $s^{\prime} \neq s$ ). The abbreviation $\sigma \mid n$ stands for the sequence of the first $n$ members of any sequence $\sigma$, finite or infinite, of elements of $\mathbb{D}$, and we write $s^{\wedge} d$ for the sequence which begins with the finite sequence $s \in \mathbb{D}^{<\omega}$ followed by $d \in \mathbb{D}$.

Recall that we use $\mathbb{N}$ for the set of positive integers and set $C=\{0,1\}^{\mathbb{N}}$ and $C_{i}=\{\iota \in C: \iota \mid n=i\}$, where $i \in\{0,1\}^{n}$.

We denote by $\pi_{X}$ and $\pi_{Y}$ the projection mappings of $X \times Y$ to $X$ and $Y$, respectively.

Lemma 3.1. Let $B$ be a Borel subset of class $\boldsymbol{\Sigma}_{\alpha}^{0}, \alpha \geq 1$, in the product $Z=X \times Y$ of a complete metric space $X$ and a Polish space $Y$. Then there are a set $\mathbb{D}$ (of sufficiently large cardinality) and sets $B_{s}, s \in \mathbb{D}^{<\omega}$, of class $\Sigma_{\alpha}^{0}$ in $Z$ such that

(a) $B_{\emptyset}=B$ and $\left\{B_{s^{\wedge} d}: d \in \mathbb{D}\right\}$ is a ( $\sigma$-discrete) cover of $B_{s}$ for $s \in \mathbb{D}^{<\omega}$ such that the family $\left\{\pi_{X}\left(B_{s^{\wedge} d}\right): d \in \mathbb{D}\right\}$ is $\sigma$-discrete;

(b) $\bigcap\left\{\overline{B_{\sigma \mid n}}: n=0,1, \ldots\right\} \subset B$ for every $\sigma \in \mathbb{D}^{\mathbb{N}}$;

(c) $\operatorname{diam} B_{s} \leq 2^{-|s|}$ for $s \in \mathbb{D}^{<\omega}$.

(We consider some fixed complete metrics on $X$ and $Y$, which are bounded by 1 , and the corresponding maximum metric on $X \times Y$.)

Proof. If $X$ is separable, the existence of such a (countable) scheme of $B$ follows from [4, Theorem 22.21] for $\alpha>1$. In the general case of $X$ and $\alpha \geq 1$, it follows from [7. Theorem $2.2(\mathrm{a}) \Rightarrow(\mathrm{b})$ ] that there is a complete sequence of $\sigma$-discrete covers $\mathcal{C}_{n}$ of $B$ by sets from $\Sigma_{\alpha}^{0}(Z)$. (We need only 
notice that $B$ is of multiplicative class $\alpha+1$ in $Z$ to get $B_{s} \in \boldsymbol{\Sigma}_{\alpha}^{0}(B)$ from the cited result. Then $B_{s} \in \Sigma_{\alpha}^{0}(Z)$ since $B \in \Sigma_{\alpha}^{0}(Z)$.) Recall that the completeness means that each filter $\mathcal{F}$ in $B$ with $\mathcal{F} \cap \mathcal{C}_{n} \neq \emptyset$ for every $n \in \mathbb{N}$ has an accumulation point, i.e., $\bigcap\{\bar{F}: F \in \mathcal{F}\} \cap B \neq \emptyset$, where the closures may be understood in $B$ or, equivalently, in $Z$. Replacing each cover by an arbitrary refinement we obtain a complete sequence of covers again. Since the cover of the metric space $Z$ by open balls of diameter at most $2^{-n}$ has a $\sigma$-discrete open refinement $\mathcal{R}_{n}$, the covers $\mathcal{C}_{n} \wedge \mathcal{R}_{n}$ are $\sigma$-discrete refinements of $\mathcal{C}_{n}$, they consist of sets of diameter at most $2^{-n}$, and their elements belong to $\boldsymbol{\Sigma}_{\alpha}^{0}(Z)$. Due to [3, Lemma 2.1], we may also achieve that even the projections of elements of each cover form a $\sigma$-discrete family of sets of the same additive class. Let $\mathcal{C}_{n}^{*}$ be such refinements of the covers $\mathcal{C}_{n} \wedge \mathcal{R}_{n}$. We can index each such cover by elements of a set $\mathbb{D}$ such that $\mathcal{C}_{n}^{*}=\left\{C_{d}^{n}: d \in \mathbb{D}\right\}$ (we can repeat some of the sets many times if the cardinality of $\mathcal{C}_{n}^{*}$ is smaller than that of $\left.\mathbb{D}\right)$. Put $B_{d_{1}, \ldots, d_{n}}=C_{d_{1}}^{1} \cap \cdots \cap C_{d_{n}}^{n}$. Note that the projections to $X$ of the finite intersections $C_{d_{1}}^{1} \cap \cdots \cap C_{d_{n}}^{n}$ are contained in the finite intersections $\pi_{X}\left(C_{d_{1}}^{1}\right) \cap \cdots \cap \pi_{X}\left(C_{d_{n}}^{n}\right)$, which form a $\sigma$-discrete family in $X$ due to our remark before Lemma 2.1. Thus (a) holds. Condition (c) is obvious. The completeness of the sequence $\left(\mathcal{C}_{n}^{*}\right)$ implies (b). The condition (b) means, due to (c), that if all the sets $B_{\sigma \mid n}, n \in \mathbb{N}$, are nonempty, then the intersection of their closures is a singleton in $B$.

Note that our requirements above are weaker than those on a Luzin scheme in [4, Theorem 22.21], in particular we do not require the injectivity of the mapping $\sigma \in \mathbb{D}^{\mathbb{N}} \mapsto \bigcap_{n \in \mathbb{N}} \overline{B_{\sigma \mid n}}$.

We need a reduction theorem for families of Borel sets of an additive class in metric spaces.

Lemma 3.2. Let $X_{a}, a \in I$, be a $\sigma$-discrete family of Borel sets of class $\boldsymbol{\Sigma}_{\alpha}^{0}, \alpha>1$, in a metric space $X$. Then there are Borel sets $X_{a}^{*} \subset X_{a}$ for $a \in I$ of class $\Sigma_{\alpha}^{0}$ in $X$ which form a partition of $\bigcup_{a \in I} X_{a}$. The same holds for $\alpha=1$ if $X$ is 0-dimensional.

Proof. For countable families this is proved in [5, $\S 30$, VII, Theorem 1 and $\S 26$, II, Theorem 1]. If $I=\bigcup_{n \in \mathbb{N}} I_{n}$ with $\left\{X_{a}: a \in I_{n}\right\}$ discrete, we may consider the family of $X(n)=\bigcup\left\{X_{a}: a \in I_{n}\right\}$, which belong to $\Sigma_{\alpha}^{0}(X)$, and choose a reduction $X^{*}(n) \subset X(n)$. Finally, put $X_{a}^{*}=X_{a} \cap X^{*}(n)$ for $a \in I_{n}$.

We are now going to prove our main uniformization theorems. The first one shows the existence of a selection, the next one shows that under a bit stronger assumptions there are continuum many disjoint uniformizations parametrized in a particular way. Although the proof of the first theorem can be understood from the second one, we prove the existence of one selec- 
tion first to point out the main idea of getting the uniformization. The same procedure will be repeated in the proof of the subsequent theorem with some more technicalities. We point out that although we prove our uniformization results for sets of an additive class in a complete metric space, we get selections defined on a metric space which need not be complete in general. We need this observation in the proof of Corollary 3.10 . We use $\widehat{X}$ to denote a completion of $X$.

Theorem 3.3. Let $X$ be a metric space and $Y$ be a Polish space. Let $\mathcal{I}_{x}, x \in X$, be $\sigma$-ideals of subsets of $Y$ such that

$$
\pi^{*}(A):=\left\{x \in X: A_{x} \notin \mathcal{I}_{x}\right\} \in \Sigma_{\alpha^{*}}^{0}(X)
$$

for all $A \subset X \times Y$ in $\boldsymbol{\Sigma}_{\alpha}^{0}(X \times Y)$, where $2 \leq \alpha \leq \alpha^{*}<\omega_{1}$ are fixed. For $X$ is 0 -dimensional, we may assume $1 \leq \alpha \leq \alpha^{*}<\omega_{1}$. Assume that

(2) $B \subset X \times Y$ is in $\boldsymbol{\Sigma}_{\alpha}^{0}(\widehat{X} \times Y)$ and $B_{x} \notin \mathcal{I}_{x}$ for every $x \in X^{*}:=\pi_{X}(B)$.

Then there is a $\boldsymbol{\Sigma}_{\alpha^{*}}^{0}(X)$-measurable selection $\xi: X^{*} \rightarrow Y$ of the mapping $x \mapsto B_{x}$. Its graph is a $\Pi_{\alpha^{*}}^{0}$-measurable uniformization of $B$ in $X \times Y$.

Proof. Fix some complete metrics on $\widehat{X}$ and $Y$ such that the diameters of $X$ and $Y$ are at most 1 and consider the maximum metric on $X \times Y$. Let $B_{s}, s \in \mathbb{D}^{<\omega}$, be a scheme of the set $B \subset \widehat{X} \times Y$ from Lemma 3.1, which exists due to assumption (2).

We are now going to define by induction a sequence of $\sigma$-discrete partitions $\mathcal{P}_{n-1}=\left\{X_{s}^{*}: s \in \mathbb{D}^{n-1}\right\}, n \in \mathbb{N}$, of $X^{*}$. We require the following properties for every $n \in \mathbb{N}$ :

(i) $\left\{X_{s^{\wedge} d}^{*}: d \in \mathbb{D}\right\}$ is a $\sigma$-discrete partition of $X_{s}^{*}$ for $s \in \mathbb{D}^{n-1}$,

(ii) $\mathcal{P}_{n-1} \subset \boldsymbol{\Sigma}_{\alpha^{*}}^{0}(X)$,

(iii) $\left(B_{s}\right)_{x} \notin \mathcal{I}_{x}$ for $x \in X_{s}^{*}$ and $s \in \mathbb{D}^{n-1}$.

Put $\mathcal{P}_{0}=\left\{X_{\emptyset}^{*}\right\}$, where $X_{\emptyset}^{*}=\pi^{*}(B)=\pi_{X}(B)$ due to (2p). Property (i) obviously holds for $\mathcal{P}_{0}$, which also satisfies (ii) and (iii) due to assumptions (1) and (2).

Given the partition $\mathcal{P}_{n-1}=\left\{X_{s}^{*}: s \in \mathbb{D}^{n-1}\right\}$ of $X^{*}$ satisfying (i) to (iii) for some $n \in \mathbb{N}$, we consider a fixed $s \in \mathbb{D}^{n-1}$. We deduce that the sets $X_{s^{\wedge} d}=X_{s}^{*} \cap \pi^{*}\left(B_{s^{\wedge} d}\right), d \in \mathbb{D}$, form a $\sigma$-discrete cover of $X_{s}^{*}$ by $\boldsymbol{\Sigma}_{\alpha^{*}}^{0}(X)$ sets, by using the fact that (iii) holds, each $\mathcal{I}_{x}$ is a $\sigma$-ideal, and the sets $\left(B_{s^{\wedge}} d\right)_{x}$, $d \in \mathbb{D}$, form a countable cover of the separable set $\left(B_{s}\right)_{x}$ (since they form a $\sigma$-discrete family, only countably many of them have a nonempty intersection with $\left.\left(B_{s}\right)_{x}\right)$. Using Lemma 3.2 , we find pairwise disjoint sets $X_{s^{\wedge} d}^{*} \subset X_{s^{\wedge} d}$, $d \in \mathbb{D}$, which cover $X_{s}^{*}$, and put $\mathcal{P}_{n}=\left\{X_{s^{\wedge} d}^{*}: s \in \mathbb{D}^{n-1}, d \in \mathbb{D}\right\}$, which is clearly $\sigma$-discrete. Thus the existence of $\mathcal{P}_{n-1}$ 's satisfying (i) to (iii) is proved. 
Now, given $x \in X^{*}$, there is a unique $s^{x} \in \mathbb{D}^{\mathbb{N}}$ such that $x \in X_{s^{x} \mid n}^{*}$ for all $n \in \mathbb{N}$. Define $\xi(x) \in \bigcap\left\{\overline{\left(B_{s^{x} \mid n}\right)_{x}}: n \in \mathbb{N}\right\}$ for $x \in X^{*}$. The definition is correct and $\xi(x) \in B_{x}$ for every $x \in X^{*}$. Indeed, the sets $\overline{\left(B_{s^{x}} \mid n\right)_{x}}, n \in \mathbb{N}$, form a descreasing sequence of nonempty (by (iii)) closed sets in $Y$ with diameter converging to zero by Lemma 3.1(c). By the completeness of the metric, the value $\xi(x)$ is uniquely defined. By property (b) of the scheme $B_{s}$, $s \in \mathbb{D}^{n}$, we get $\xi(x) \in B_{x}$. To verify that $\xi$ is $\boldsymbol{\Sigma}_{\alpha^{*}}^{0}(X)$-measurable, define $\xi_{n}$ to be constant on each $X_{s}^{*}, s \in \mathbb{D}^{n}$. Let, e.g., $\xi_{n}(x)$ for $x \in X_{s}^{*}\left(=X_{s^{x} \mid n}^{*}\right)$ be an arbitrary element of the projection of $B_{s}\left(=B_{s^{x} \mid n}\right)$ to $Y$. Then each $\xi_{n}$ is $\boldsymbol{\Sigma}_{\alpha^{*}}^{0}(X)$-measurable and it converges uniformly to $\xi$ due to the condition on the diameters of $B_{s}$ from Lemma 3.1(c). So $\xi$ is $\Sigma_{\alpha^{*}}^{0}(X)$-measurable as well (see [5, $\S 31$, VIII, Theorem 2]). The graph of $\xi$ is in $\mathbf{\Pi}_{\alpha^{*}}^{0}$ by [5, $\S 31$, VII, Theorem 1]. This concludes the proof.

The next theorem is a strengthening of the previous one under the assumption that the $\sigma$-ideals $\mathcal{I}_{x}$ contain singletons. It is inspired by and might be compared with Mauldin's [8, Theorem 1.1].

Theorem 3.4. Let $X, Y, \mathcal{I}_{x}$ for $x \in X$, and $B \subset X \times Y$ satisfy the assumptions of Theorem 3.3. If, moreover, $\mathcal{I}_{x}$ contains all singletons in $B_{x}$ for each $x \in X$, then there is a Borel isomorphism $\Xi$ of $X^{*} \times C$ onto $R \subset B$, with $X^{*}:=\pi_{X}(B)$, which is of the form $\Xi(x, \iota)=(x, \Phi(x, \iota))$, such that

(a) $\Phi(\cdot, \iota)$ is a $\boldsymbol{\Sigma}_{\alpha^{*}}^{0}(X)$-measurable selection of $x \in X^{*} \mapsto B_{x}$ for every $\iota \in C$;

(b) $\Phi(x, \cdot)$ is a homeomorphism of $C$ onto $R_{x}$ for every $x \in X^{*}$;

(c) $\Xi: X^{*} \times C \rightarrow R$ is $\Sigma_{\alpha^{*}}^{0}(X \times Y)$-measurable and $\Xi^{-1}: R \rightarrow X \times C$ is $\Sigma_{\alpha^{*}}^{0}(R)$-measurable;

(d) $F: x \in X^{*} \mapsto R_{x}$ is both upper and lower $\Sigma_{\alpha^{*}}^{0}(X)$-measurable;

(e) $R=\Xi\left(X^{*} \times C\right)$ is in $\Pi_{\alpha^{*}}^{0}(X \times Y)$.

Proof. The notions of distance and diameter are related to the maximum metric, defined using complete metrics on $\widehat{X}$ and $Y$ giving diameter less than one as in the proof of Theorem 3.3 . Let $B_{s}, s \in \mathbb{D}^{<\omega}$, be a scheme of the set $B \subset X \times Y$ from Lemma 3.1 which exists due to assumption (2).

We are now going to define a sequence of partitions of $X^{*}$ by induction. The elements of the $n$th partition will be indexed by the elements of the set $\mathcal{T}_{n}$ of mappings ("strategies") $\tau$ from $\{0,1\}^{\leq n}$ to $\mathbb{D}^{<\omega}$ such that

(A) $\tau\left(i^{\wedge} j\right) \succ \tau(i)$ for $i \in\{0,1\}^{<n}$ and $j \in\{0,1\}$ if $n \in \mathbb{N}$,

(B) the sets $\overline{B_{\tau(i)}}, i \in\{0,1\}^{n}$, are pairwise disjoint.

We write $\mathcal{T}_{0}$ for the singleton which contains just the mapping $\tau_{0}: \emptyset \rightarrow \emptyset$. The elements of the $n$th partition will be denoted by $X_{n}^{*}(\tau)$, where $\tau \in \mathcal{T}_{n}$. We denote by $\mathcal{T}_{n}(\tau)$ the set of elements of $\mathcal{T}_{n}$ which extend $\tau \in \mathcal{T}_{n-1}$, i.e., 


$$
\mathcal{T}_{n}(\tau):=\left\{\tau^{\prime} \in \mathcal{T}_{n}: \tau^{\prime} \uparrow\{0,1\} \leq(n-1)=\tau\right\}
$$

for $n \in \mathbb{N}$. We require the following properties for every $\tau \in \mathcal{T}_{n}$ and $n=$ $0,1, \ldots$ :

(i) each $X_{n}^{*}(\tau)$ is in $\boldsymbol{\Sigma}_{\alpha^{*}}^{0}(X)$;

(ii) $X_{n}^{*}(\tau)=\bigcup\left\{X_{n+1}^{*}\left(\tau^{\prime}\right): \tau^{\prime} \in \mathcal{T}_{n+1}(\tau)\right\}$;

(iii) $\left(B_{\tau(i)}\right)_{x} \notin \mathcal{I}_{x}$ for $x \in X_{n}^{*}(\tau)$ and $i \in\{0,1\}^{n}$.

Put $X_{0}^{*}\left(\tau_{0}\right)=\pi^{*}(B)$ (cf. (1) from Theorem 3.3 for the notation). It is in $\boldsymbol{\Sigma}_{\alpha^{*}}^{0}(X)$ and it is equal to $X^{*}$ by our assumptions (1) and (2). Thus the partition $\left\{X_{0}^{*}\left(\tau_{0}\right)\right\}$ of $X^{*}$ satisfies (i) and (iii). Condition (ii) requires nothing for $n=0$.

Given the partition $\left\{X_{n-1}^{*}(\tau): \tau \in \mathcal{T}_{n-1}\right\}$ satisfying (i) to (iii) for some $n \in \mathbb{N}$, we will consider a cover of each $X_{n-1}^{*}(\tau)$ with $\tau \in \mathcal{T}_{n-1}$ fixed. For every $\tau^{\prime} \in \mathcal{T}_{n}(\tau)$, put

$$
X_{n}\left(\tau^{\prime}\right)=X_{n-1}^{*}(\tau) \cap \bigcap\left\{\pi^{*}\left(B_{\tau^{\prime}(i)}\right): i \in\{0,1\}^{n}\right\} .
$$

Observe that the family $\left\{X_{n}\left(\tau^{\prime}\right): \tau^{\prime} \in \mathcal{T}_{n}(\tau)\right\}$ is a cover of $X_{n-1}^{*}(\tau)$ for every $\tau \in \mathcal{T}_{n-1}$. Indeed, let $x \in X_{n-1}^{*}(\tau)$. By (iii) and (B), $\left(B_{\tau(i)}\right)_{x} \notin \mathcal{I}_{x}$ for every $i \in\{0,1\}^{n-1}$ and the sets $\overline{B_{\tau(i)}}, i \in\{0,1\}^{n-1}$, are pairwise disjoint. Since $\left(B_{\tau(i)}\right)_{x} \notin \mathcal{I}_{x}$, there are two distinct points $y_{0}(x, i), y_{1}(x, i) \in$ $\left(B_{\tau(i)}\right)_{x}$, for each fixed $i \in\{0,1\}^{n-1}$, such that $U \cap\left(B_{\tau(i)}\right)_{x} \notin \mathcal{I}_{x}$ for every neighborhood $U$ of $y_{0}(x, i)$, and $y_{1}(x, i)$, respectively. (Otherwise, if there is at most one such point, there would be a cover of the separable metric space $\left(B_{\tau(i)}\right)_{x}$ by countably many elements of $\mathcal{I}_{x}$, namely countably many neighbourhoods of some points in $\left(B_{\tau(i)}\right)_{x}$ and at most one singleton in $B_{x}$, a contradiction with $\left(B_{\tau(i)}\right)_{x} \notin \mathcal{I}_{x}$. Here we use the extra assumption on $\mathcal{I}_{x}$.) Choose open neighborhoods $U_{j}(x, i)$ of $y_{j}(x, i)$ for $j=0,1$ of diameters less than $\frac{1}{3} \operatorname{dist}\left(y_{0}(x, i), y_{1}(x, i)\right)$. Since the sets $\left(B_{\tau(i)}\right)_{x} \cap U_{j}(x, i), j=0,1$, are not in $\mathcal{I}_{x}$ and they are covered by the countably many sets $\left(B_{s}\right)_{x} \subset\left(B_{\tau(i)}\right)_{x}$ with $s \succ \tau(i)$ for which $\left(B_{s}\right)_{x} \subset$ $U_{j}(x, i)$ and $2^{-|s|} \leq \frac{1}{3} \operatorname{dist}\left(y_{0}(x, i), y_{1}(x, i)\right)$ by Lemma 3.1 (a) \& (c), we find $\tau_{x}\left(i^{\wedge} j\right) \succ \tau(i)$ such that $2^{-\left|\tau_{x}\left(i^{\wedge} j\right)\right|} \leq \frac{1}{3} \operatorname{dist}\left(y_{0}(x, i), y_{1}(x, i)\right),\left(B_{\tau_{x}\left(i^{\wedge} j\right)}\right)_{x} \subset$ $U_{j}(x, i)$, and $\left(B_{\tau_{x}\left(i^{\wedge} j\right)}\right)_{x} \notin \mathcal{I}_{x}$ for $j=0,1$. The sets $\overline{B_{\tau_{x}\left(i^{\wedge} j\right)}}$ are disjoint, since $\overline{B_{\tau_{x}\left(i^{\wedge} j\right)}} \subset \overline{B_{\tau(i)}}$, the sets $\overline{B_{\tau(i)}}$ form a pairwise disjoint family, and $\operatorname{dist}\left(\overline{B_{\tau_{x}\left(i^{\wedge} 0\right)}}, \overline{B_{\tau_{x}\left(i^{\wedge} 1\right)}}\right) \geq \frac{1}{3} \operatorname{dist}\left(y_{0}(x, i), y_{1}(x, i)\right)>0$. Put $\tau_{x}\left\lceil\{0,1\}^{(n-1)}=\tau\right.$. Now $x \in X_{n}\left(\tau_{x}\right)$ and $\tau_{x} \in \mathcal{T}_{n}(\tau)$.

Let us check that $X_{n}\left(\tau^{\prime}\right) \in \boldsymbol{\Sigma}_{\alpha^{*}}^{0}(X)$ for all $\tau^{\prime} \in \mathcal{T}_{n}$. As $X_{n-1}^{*}(\tau) \in \boldsymbol{\Sigma}_{\alpha^{*}}^{0}(X)$ for $\tau \in \mathcal{T}_{n-1}$ by (i), we see that each $X_{n}\left(\tau^{\prime}\right)=X_{n-1}^{*}(\tau) \cap \bigcap\left\{\pi^{*}\left(B_{\tau^{\prime}(i)}\right): i \in\right.$ $\left.\{0,1\}^{n}\right\}$ is in $\boldsymbol{\Sigma}_{\alpha^{*}}^{0}(X)$ as a finite intersection of elements of $\boldsymbol{\Sigma}_{\alpha^{*}}^{0}(X)$ by 11 for $\tau^{\prime} \in \mathcal{T}_{n}(\tau)$. 
By Lemma 3.1(a) the family $\mathcal{D}=\left\{\pi\left(B_{s}\right): s \in \mathbb{D}^{<\omega}\right\}$ is $\sigma$-discrete (it is not difficult to show by induction that all the families $\left\{\pi\left(B_{s}\right): s \in \mathbb{D}^{n}\right\}$ are $\sigma$-discrete using (a) and realize that $\mathcal{D}$ is their countable union). Since $\pi^{*}\left(B_{s}\right) \subset \pi\left(B_{s}\right)$, also the family $\mathcal{D}^{*}=\left\{\pi^{*}\left(B_{s}\right): s \in \mathbb{D}^{<\omega}\right\}$ is $\sigma$-discrete. Therefore also $\mathcal{D}_{1}=\mathcal{D}^{*}, \mathcal{D}_{2}=\mathcal{D}^{*} \wedge \mathcal{D}^{*}, \mathcal{D}_{3}=\mathcal{D}^{*} \wedge \mathcal{D}^{*} \wedge \mathcal{D}^{*}, \ldots$ are $\sigma$-discrete, as also is their (countable) union $\mathcal{E}=\bigcup_{n \in \mathbb{N}} \mathcal{D}_{n}$. The family $\left\{\bigcap\left\{\pi^{*}\left(B_{\tau^{\prime}(i)}\right)\right.\right.$ : $\left.\left.i \in\{0,1\}^{n}\right\}: \tau^{\prime} \in \mathcal{T}_{n}(\tau)\right\}$ is $\sigma$-discrete as a subfamily of $\mathcal{E}$. Finally, the family $\left\{X_{n-1}^{*}(\tau) \cap \bigcap\left\{\pi^{*}\left(B_{\tau^{\prime}(i)}\right): i \in\{0,1\}^{n}\right\}: \tau^{\prime} \in \mathcal{T}_{n}(\tau)\right\}$ is $\sigma$-discrete. We have proved above that it is a cover of $X_{n-1}^{*}(\tau)$.

Hence we can find a $\sigma$-discrete partition $\left\{X_{n}^{*}\left(\tau^{\prime}\right): \tau^{\prime} \in \mathcal{T}_{n}(\tau)\right\}$ of $X_{n-1}^{*}(\tau)$ consisting of elements of $\boldsymbol{\Sigma}_{\alpha^{*}}^{0}(X)$ by applying Lemma 3.2 to the family $\left\{X_{n}\left(\tau^{\prime}\right): \tau^{\prime} \in \mathcal{T}_{n}(\tau)\right\}$.

Thus for every $\tau \in \mathcal{T}_{n}$ we have an $X_{n}^{*}(\tau)$ such that conditions (i)-(iii) are satisfied.

The requirement that $x \in X_{n}^{*}(\tau)$ defines uniquely a $\tau=\tau_{n}^{x} \in \mathcal{T}_{n}$ for $x \in X^{*}$. Due to (ii) there is a unique $\tau^{x}:\{0,1\}^{<\omega} \rightarrow \mathbb{D}^{<\omega}$ with $\tau_{n}^{x}=\tau^{x}\left\{\{0,1\}^{\leq n}\right.$ for every $n=0,1, \ldots$ We define the required mapping $\Xi: X \times C \rightarrow B$ by $\Xi(x, \iota) \in \bigcap_{n=0}^{\infty} \overline{B_{\tau^{x}(\iota \mid n)}}$. This indeed defines a mapping of $X \times C$ to $B$, because the sets $B_{\tau^{x}(\iota \mid n)}$ are decreasing and nonempty (by (iii)) with $\bigcap_{n=0}^{\infty} \overline{B_{\tau^{x}(\iota \mid n)}}$ a singleton in $B$ by properties (b) and (c) of our scheme from Lemma 3.1. Moreover, all the sets $B_{\tau^{x}(\iota \mid n)}$ have nonempty $x$-section $\left(B_{\tau^{x}(\iota \mid n)}\right)_{x}$ by (iii), i.e., the intersection of $B_{\tau^{x}(\iota \mid n)}$ with the closed set $\{x\} \times Y \subset X \times Y$ is nonempty, and so $\bigcap_{n \in \mathbb{N}} \overline{B_{\tau^{x}(\iota \mid n)}} \cap(\{x\} \times$ $Y) \neq \emptyset$. Thus this intersection is the singleton $\{\Xi(x, \iota)\}=\bigcap_{n \in \mathbb{N}} \overline{B_{\tau^{x}(\iota \mid n)}}$, and $\Xi(x, \iota) \in\{x\} \times B_{x}$. So $\Xi$ is of the form $\Xi(x, \iota)=(x, \Phi(x, \iota))$ with $\Phi: X \times C \rightarrow Y$ uniquely determined by $\Phi(x, \iota) \in \bigcap_{n \in \mathbb{N}} \overline{\left(B_{\left.\tau^{x}(\iota \mid n)\right)_{x}}\right.}=$ $\left(\bigcap_{n \in \mathbb{N}} \overline{B_{\tau^{x}(\iota \mid n)}}\right)_{x}$.

It remains to show that $\Xi$ has the required properties (a)-(e).

We prove (a) by constructing a sequence of $\boldsymbol{\Sigma}_{\alpha^{*}}^{0}$-measurable mappings $\Phi_{n}$ which converges uniformly to $\Phi$. We define $\Phi_{n}$ to take a constant value from $\pi_{Y}\left(B_{\tau(i)}\right)$ on $X_{n}^{*}(\tau) \times C_{i}$ for every $\tau \in \mathcal{T}_{n}$ and $i \in\{0,1\}^{n}$. This is possible since $\left(B_{\tau(i)}\right)_{x} \notin \mathcal{I}_{x}$ if $x \in X_{n}^{*}(\tau)$ by (iii) and so $B_{\tau(i)} \neq \emptyset$.

Let $W$ be open in $Y$. Then

$$
\Phi_{n}^{-1}(W)=\bigcup\left\{X_{n}^{*}(\tau) \times C_{i}: \tau \in \mathcal{T}_{n}, i \in\{0,1\}^{n}, \Phi_{n}\left(X_{n}^{*}(\tau) \times C_{i}\right) \subset W\right\},
$$

because $\Phi_{n}$ is constant on each $X_{n}^{*}(\tau) \times C_{i}$. Since the $\sigma$-discrete union of sets $X_{n}^{*}(\tau) \times C_{i}$ over any subset of pairs of $\tau \in \mathcal{T}_{n}$ and $i \in\{0,1\}^{n}$ is in $\boldsymbol{\Sigma}_{\alpha^{*}}^{0}(X \times C)$, the mapping $\Phi_{n}$ is $\boldsymbol{\Sigma}_{\alpha^{*}}^{0}(X \times C)$-measurable. The mappings $\Phi_{n}$ converge to $\Phi$ uniformly (in both $x$ and $\iota$ ) since the diameter of each $\pi_{Y}\left(B_{\tau(i)}\right)$ is at most $2^{-n}$. By [5, §31, VIII, Theorem 2], $\Phi$ is also $\boldsymbol{\Sigma}_{\alpha^{*}}^{0}(X \times C)$-measurable. In particular, (a) is proved. 
The mapping $\Phi(x, \cdot)$ is one-to-one and continuous since $\overline{B_{\tau^{x}(i)}} \cap \overline{B_{\tau^{x}\left(i^{\prime}\right)}}$ $=\emptyset$ for $i \neq i^{\prime}, i, i^{\prime} \in\{0,1\}^{n}$, and since $\operatorname{diam} \pi_{Y}\left(B_{\tau^{x}(i)}\right) \leq 2^{-n}$ for $i \in\{0,1\}^{n}$. Hence (b) follows by the compactness of $C$.

Let $U$ be open in $X$ and $W$ be open in $Y$. Then $\Xi^{-1}(U \times W)=(U \times$ $Y) \cap \Phi^{-1}(W)$. Thus, to prove that $\Xi$ is $\boldsymbol{\Sigma}_{\alpha^{*}}^{0}(X \times C)$-measurable, it is enough to show that $\Phi$ is $\boldsymbol{\Sigma}_{\alpha^{*}}^{0}(X \times C)$-measurable. However, this was proved above. So the first claim of (c) is verified.

We see that $\Xi$ is injective by (b). To show that $\Xi^{-1}$ is $\Sigma_{\alpha^{*}}^{0}(R)$-measurable, note that $\Xi\left(U \times C_{i}\right)=(U \times Y) \cap \Xi\left(X^{*} \times C_{i}\right)$ for $U$ open in $X$ and $i \in\{0,1\}^{n}$. Thus we need to prove that $\Xi\left(X^{*} \times C_{i}\right)$ is in $\Sigma_{\alpha^{*}}^{0}$ in $R$ for each $n \in \mathbb{N}$ and $i \in\{0,1\}^{n}$. The point $(x, y) \in R$ belongs to $\Xi\left(X^{*} \times C_{i}\right)$ if and only if $(x, y) \in \overline{B_{\tau^{x}(i)}}$ and $x \in X_{n}^{*}\left(\tau_{n}^{x}\right)$. Thus $\Xi\left(X^{*} \times C_{i}\right)=R \cap \bigcup\left\{\left(X_{n}^{*}(\tau)\right) \times Y\right)$ $\left.\cap \overline{B_{\tau(i)}}: \tau \in \mathcal{T}_{n}\right\}$. This is the union of a $\sigma$-discrete family of elements of $\boldsymbol{\Sigma}_{\alpha^{*}}^{0}(X \times Y)$ intersected with $R$, and so an element of $\boldsymbol{\Sigma}_{\alpha^{*}}^{0}$ in $R$. This concludes the verification of (c).

Let $W \subset Y$ be open and nonempty. If $x \in F^{-1}(W)$ then there is a $y=\Phi(x, \iota) \in W$ for some $\iota \in C$. Thus the distance $\delta$ of $y$ and $W^{c}$ is positive and $\{y\}=\bigcap_{n \in \mathbb{N}}\left(\overline{B_{\tau^{x}(\iota \mid n)}}\right)_{x} \subset W$. So there is an $n \in \mathbb{N}$ such that the diameter of $B_{\tau^{x}(\iota \mid n)}$ is less than $\delta$ and $(x, y) \in \overline{B_{\tau^{x}(\iota \mid n)}} \subset X \times W$. Having $n$ and $\iota$ such that $\overline{B_{\tau^{x}(\iota \mid n)}} \subset X \times W$, we have $\{y\}=\bigcap_{n \in \mathbb{N}}\left(\overline{B_{\tau^{x}(\iota \mid n)}}\right)_{x} \subset W$, and thus $y \in W$. Therefore $x \in F^{-1}(W)$ if and only if there are $n \in \mathbb{N}$ and $i \in\{0,1\}^{n}$ such that $\pi_{Y}\left(\overline{B_{\tau^{x}(i)}}\right) \subset W$. Thus

$$
F^{-1}(W)=\bigcup\left\{X_{n}^{*}(\tau): n \in \mathbb{N}, \tau \in \mathcal{T}_{n}, i \in\{0,1\}^{n}, \pi_{Y}\left(\overline{B_{\tau(i)}}\right) \subset W\right\} .
$$

So $F$ is lower $\boldsymbol{\Sigma}_{\alpha^{*}}^{0}(X)$-measurable.

Let the compact set $\Phi(x, C)$ be a subset of $W$. Thus it has a positive distance from $W^{c}$ and so $\overline{B_{\tau^{x}(\iota \mid n)}} \subset W$ for sufficiently large $n \in \mathbb{N}$ and every $\iota \in C$. Conversely, if the latter condition holds, then $\Phi(x, C) \subset W$. Thus

$$
F_{-1}(W)=\bigcup\left\{X_{n}^{*}(\tau): n \in \mathbb{N}, \tau \in \mathcal{T}_{n}, \pi_{Y}\left(\overline{B_{\tau(i)}}\right) \subset W \text { for every } i \in\{0,1\}^{n}\right\} .
$$

This is again a $\sigma$-discrete union of elements of $\boldsymbol{\Sigma}_{\alpha^{*}}^{0}(X)$ and so $F$ is also upper $\Sigma_{\alpha^{*}}^{0}(X)$-measurable, and $(\mathrm{d})$ is proved.

Let $\mathcal{W}$ be a countable base for the topology of $Y$. We can easily check that $(x, y) \notin R$ if and only if there is a $W \in \mathcal{W}$ such that $y \in W, F(x) \subset$ $Y \backslash \bar{W}$ since $F(x)$ is closed. As $F$ is upper $\boldsymbol{\Sigma}_{\alpha^{*}}^{0}(X)$-measurable, the set $R^{c}=\bigcup_{W \in \mathcal{W}} F_{-1}(Y \backslash \bar{W}) \times W$ is in $\Sigma_{\alpha^{*}}^{0}(X \times Y)$, and (e) is also proved.

As corollaries of Theorems 3.3 and 3.4 , and Lemmas 2.1 and 2.2 on the "generalized projections" $\pi_{\mu}^{*}$ and $\pi_{F}^{*}$ from the previous section, we get the following results. 
Theorem 3.5. Let $X$ be a metrizable space, $Y$ be a Polish space, and $\mu: X \times \mathcal{B}(Y) \rightarrow[0,1]$ be such that

(a) $\mu(x, \cdot)$ is a Borel probability on $Y$ for every $x \in X$,

(b) $\{x \in X: \mu(\cdot, H)>r\}$ is in $\Sigma_{\alpha_{0}}^{0}(X)\left(1 \leq \alpha_{0}<\omega_{1}\right)$ for every open $H \subset Y$ and $r \in \mathbb{R}$.

Let $B \subset X \times Y$ be in $\boldsymbol{\Sigma}_{\alpha}^{0}(\widehat{X} \times Y)$ for some $\alpha$ with $2 \leq \alpha<\omega_{1}$, or $1 \leq \alpha$ $<\omega_{1}$ if $X$ is 0 -dimensional. Let $\mu\left(x, B_{x}\right)>0$ for every $x \in \pi_{X}(B)$. Let $\alpha^{*}$ be as in Lemma 2.1. In particular, if $\mu(x, \cdot)=\mu$, then $\alpha^{*}=\alpha$.

Then there is a $\boldsymbol{\Sigma}_{\alpha^{*}}^{0}(X)$-measurable mapping $\xi: \pi_{X}(B) \rightarrow Y$ whose

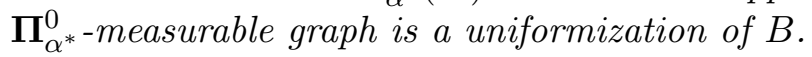

If, moreover,

(c) $\mu(x, \cdot)$ does not have atoms for every $x \in X$, then there is a mapping $\Xi: \pi_{X}(B) \times Y \rightarrow B$ as in Theorem 3.4 .

Proof. Put $\mathcal{I}_{x}=\left\{N \subset B_{x}: \mu(x, N)=0\right\}$ for every $x \in X$. By Lemma 2.1, we have $\pi^{*}(A)=\pi_{\mu}^{*}(A, 0) \in \boldsymbol{\Sigma}_{\alpha^{*}}^{0}(X)$ for every $A \in \boldsymbol{\Sigma}_{\alpha}^{0}(X \times Y)$, so we may apply Theorems 3.3 and 3.4 .

Theorem 3.6. Let $X$ be a metrizable space and $Y$ be a Polish space. Let $F: X \rightarrow \mathcal{P}(Y)$ be such that

(a) $F(x)$ is a Baire subspace of $Y$ for every $x \in X$,

(b) $F$ is lower $\boldsymbol{\Sigma}_{\alpha_{0}}^{0}$-measurable $\left(1 \leq \alpha_{0}<\omega_{1}\right)$.

Let $B \subset \operatorname{graph} F$ be in $\Sigma_{\alpha}^{0}(\widehat{X} \times Y)$ for some $\alpha$ with $2 \leq \alpha<\omega_{1}$, or $1 \leq$ $\alpha<\omega_{1}$ if $X$ 0-dimensional. Let $B_{x}$ be nonmeager in $F(x)$ for $x \in \pi_{X}(B)$. Let $\alpha^{*}$ be as in Lemma 2.1. In particular, if $F(x)=Y$, then $\alpha^{*}=\alpha$.

Then there is a $\boldsymbol{\Sigma}_{\alpha^{*}}^{0}(X)$-measurable mapping $\xi: \pi_{X}(B) \rightarrow Y$ whose $\Pi_{\alpha^{*}}^{0}$-measurable graph is a uniformization of $B$.

If moreover

(c) $F(x)$ has no isolated point for every $x \in X$, then there is a mapping $\Xi: \pi_{X}(B) \times C \rightarrow B$ as in Theorem 3.4 .

Proof. Put $\mathcal{I}_{x}=\left\{N \subset B_{x}: N\right.$ is meager in $\left.B_{x}\right\}$ for $x \in X$. Now $\pi^{*}(A)=\pi_{F}^{*}(A, Y) \in \boldsymbol{\Sigma}_{\alpha^{*}}^{0}(X)$ for every $A \in \boldsymbol{\Sigma}_{\alpha}^{0}(X \times Y)$ by Lemma 2.2 . Finally, we apply Theorems 3.3 and 3.4 .

As a particular case, we point out a refinement of the theorem of Srivastava for uniformizations of Borel sets with $G_{\delta}$ sections ([12, Theorem 4.1]).

Corollary 3.7. Let $X$ be a metric space and $Y$ a Polish space. Assume that $B \subset X \times Y$ is a Borel subset of class $\Sigma_{\alpha}^{0}$ in $\widehat{X} \times Y$ for some $\alpha$ with $2 \leq$ $\alpha<\omega_{1}$, or $1 \leq \alpha<\omega_{1}$ if $X$ is 0 -dimensional. Let the sections $B_{x}, x \in X$, be 
$G_{\delta}$ in $Y$ and the mapping $F: x \mapsto B_{x}$ be lower $\boldsymbol{\Sigma}_{\alpha_{0}}^{0}(X)$-measurable. Then there is a $\boldsymbol{\Sigma}_{\alpha^{*}}^{0}(X)$-measurable selection of $F$, where $\alpha^{*}$ is as in Lemma 2.1.

Proof. As $G_{\delta}$ subsets of the Polish space $Y$, the sets $B_{x}$ are Baire spaces and, in particular, they are not meager in themselves for $x \in \pi_{X}(B)$. So we may put $F(x)=B_{x}$ and apply Theorem 3.6 .

We show in the following example that the class of the selection mapping $\xi$ in Theorems 3.5 and 3.6 cannot be improved, even its graph (the uniformization) cannot be of a lower multiplicative class for sets of nonlimit ambiguous class higher than 1.

EXAmple 3.8. There is a $\boldsymbol{\Delta}_{\alpha+1}^{0}$ subset $B$ of $\mathbb{R}^{2}$ for every $\alpha \in\left[1, \omega_{1}\right)$ such that $\lambda\left(B_{x}\right)>0$ and $B_{x}$ is nonmeager for every $x \in \mathbb{R}$, but there is no $\Pi_{\alpha}^{0}$ uniformization of $B$.

Proof. We use the notation $X=Y=Z=\mathbb{R}$. Let $\alpha \in\left[1, \omega_{1}\right)$.

Let $U \subset X \times(Y \times Z)$ be a $\Pi_{\alpha}^{0}$ universal set for $\Pi_{\alpha}^{0}$ sets in $Y \times Z$ (e.g., $U \subset C \times(Y \times Z)$ in $\Pi_{\alpha}^{0}(C \times Y \times Z)$ by [4, Theorem 22.3] is $\Pi_{\alpha}^{0}$ in $X \times Y \times Z$ as well).

Let $A=\left\{(x, x) \in X \times Y: \lambda\left(U_{(x, x)}^{c}\right)>0\right.$ and $U_{(x, x)}^{c}$ is nonmeager in $\left.\mathbb{R}\right\}$. It is $\Sigma_{\alpha}^{0}$ in $D=\{(x, x): x \in X\}$ by the lemmas on generalized projections. Put $B=([A \times Z] \backslash U) \cup([D \backslash A] \times Z)$. Then $B$ is in $\Delta_{\alpha+1}^{0}(D \times Z)$ and $\lambda\left(B_{(x, x)}\right)>0$ and $B_{(x, x)}$ is nonmeagre for every $x \in \mathbb{R}$ by the definition of $B$.

Let $G$ be a uniformization of $B$ in $\Pi_{\alpha}^{0}(D \times Z)$. By the definition of a uniformization, $G_{(x, x)} \neq \emptyset$ for every $(x, x) \in D$ since $B_{(x, x)} \neq \emptyset$ for every $(x, x) \in D$.

Since $U$ is universal, there is $x \in X$ such that $U_{x}=\pi_{Y \times Z}(G)$. So $U_{(x, y)}=$ $G_{(y, y)}$ for every $y \in Y$, and in particular $U_{(x, x)}=G_{(x, x)}$ is a singleton. Thus $(x, x) \in A$. Therefore $G_{(x, x)} \subset B_{(x, x)} \subset U_{(x, x)}^{c}$. It follows that $\emptyset \neq G_{(x, x)}=$ $U_{(x, x)} \subset U_{(x, x)}^{c}$, a contradiction.

As $D$ can be identified with $\mathbb{R}, B$ is our example.

We still give a trivial example showing that we cannot replace the additive class $\boldsymbol{\Sigma}_{\alpha}^{0}$ of $B$ in Theorems 3.5 and 3.6 by the multiplicative class $\boldsymbol{\Pi}_{\alpha}^{0}$ to get a uniformization of class $\boldsymbol{\Pi}_{\alpha^{*}}^{0}$.

Example 3.9. Let $X=\{0\} \cup\{1 / n: n \in \mathbb{N}\}, Y=[-2,2]$. The set $B=\left\{(1 / n, y) \in X \times Y: n \in \mathbb{N}, y \in\left[(-1)^{n}-1 / n,(-1)^{n}+1 / n\right]\right\} \cup$ $(\{0\} \times[-1,1])$, is closed, the space $X$ is 0 -dimensional, and there is no closed uniformization. The sets $B_{x}$ are intervals in $Y$, so they are of positive measure $\mu$ and nonmeager in $Y$.

Our result also gives an estimate, probably not the best possible one, on the Borel class for selectors of partitions into "relatively large sets". The first 
assumption (a) gives an improvement of Srivastava's theorem on selectors for partitions into $G_{\delta}$ sets (see [12, Theorem 5.1]).

Corollary 3.10. Let $Y$ be a Polish space and $\mathcal{P}$ be a partition of $Y$ such that either

(a) $P$ is a nonempty set which is residual in $\bar{P}$ for every $P \in \mathcal{P}$, or

(b) $\mu(P)>\frac{1}{2} \mu(\bar{P})$ for every $P \in \mathcal{P}$, with respect to some fixed Borel probability on $Y$,

and the set $W^{*}:=\bigcup\{P \in \mathcal{P}: P \cap W \neq \emptyset\}$ is in $\boldsymbol{\Delta}_{\alpha}^{0}$ for every $W \in \mathcal{W}$, where $\mathcal{W}$ is a countable open base of $Y$ and $1 \leq \alpha<\omega_{1}$ is fixed. Then there is a selector mapping $s: Y \rightarrow Y(s(P)=\{y\} \subset P$, for every $P \in \mathcal{P})$ which is $\boldsymbol{\Sigma}_{\alpha+\alpha+1}^{0}$-measurable. There is also a selector set $S \subset Y$ (the cardinality of each $S \cap P$ for $P \in \mathcal{P}$ is one) of class $\Pi_{\alpha+\alpha+1}^{0}$.

Proof. Let $\mathcal{W}=\left\{W_{n}: n \in \mathbb{N}\right\}$. Let $f: Y \rightarrow C=\{0,1\}^{\mathbb{N}}$ be the characteristic function of the family $\left(W_{n}^{*}: n \in \mathbb{N}\right)$, i.e., $f(y)=\left(i_{1}, i_{2}, \ldots\right)$, where $i_{n}=1$ if and only if $y \in W_{n}^{*}$. This mapping is clearly $\boldsymbol{\Sigma}_{\alpha}^{0}$-measurable, so its graph is in $\boldsymbol{\Pi}_{\alpha}^{0}(Y \times C)$, and thus also in $\boldsymbol{\Sigma}_{\alpha+1}^{0}(Y \times C)$. We observe that the following claim holds:

Claim. The equality $f(y)=f\left(y^{\prime}\right)$ is equivalent to $y$ and $y^{\prime}$ lying in the same element of $\mathcal{P}$.

Indeed, if $y, y^{\prime} \in P \in \mathcal{P}$, then $f(y)=f\left(y^{\prime}\right)$. If $y \in P \in \mathcal{P}$ and $y^{\prime} \in$ $P^{\prime} \in \mathcal{P}$, where $P \neq P^{\prime}$, then $P^{\prime} \backslash \bar{P} \neq \emptyset$ or $P \backslash \overline{P^{\prime}} \neq \emptyset$ since otherwise $P$ or $P^{\prime}$ is meager in $\bar{P}=\overline{P^{\prime}}$ in case (a), and $P$ or $P^{\prime}$ is of measure at most $\frac{1}{2} \mu(\bar{P})=\frac{1}{2} \mu\left(\overline{P^{\prime}}\right)$ in case (b), which contradicts (a) and (b), respectively. Thus there is a $W_{n} \in \mathcal{W}$ such that $P^{\prime} \subset W_{n}^{*}$ and $P \cap W_{n}^{*}=\emptyset$, or vice versa. This implies that $f(y)$ and $f\left(y^{\prime}\right)$ have distinct $n$th coordinates.

To prove case (a) we consider the inverse $f^{-1}: f(Y) \rightarrow \mathcal{P}(Y)$. It is a multivalued mapping which is lower semicontinuous (lower $\boldsymbol{\Sigma}_{1}^{0}$-measurable) on $X:=f(Y)$ because $f\left(W_{n}\right)=f\left(W_{n}^{*}\right)=\left\{\iota \in f(Y): \iota_{n}=1\right\}$ for every $n \in \mathbb{N}$. This follows easily from the Claim. Thus the multivalued mapping $F: X \rightarrow \mathcal{P}(Y)$ defined by $F(x)=\overline{f^{-1}(x)}$ is also lower semicontinuous on $X$. The subspaces $F(x)$ are closed (and nonempty) in the Polish space $Y$ for $x \in X$, so they are Baire spaces. Let $\mathcal{I}_{x}$ denote the $\sigma$-ideal of meager subsets of $F(x)$ for every $x \in X$. Lemma 2.2 implies that condition (1) of Theorem 3.3 holds for every $1 \leq \alpha=\alpha^{*}<\omega_{1}$.

We put $B=\operatorname{graph} f^{-1}$. It is in $\boldsymbol{\Sigma}_{\alpha+1}^{0}(C \times Y)$ since graph $f \in \boldsymbol{\Sigma}_{\alpha+1}^{0}(Y \times C)$. The sets $B_{x}=f^{-1}(x)$ are nonempty residual in $F(x)$ for every $x \in X$ by (a). So assumption (2) of Theorem 3.3 is satisfied with $\alpha+1$ instead of $\alpha$.

To prove case (b) put $B=$ graph $f^{-1} \in \Sigma_{\alpha+1}^{0}(C \times Y)$ again and let $\mathcal{I}_{x}=\mathcal{I}$ be the $\sigma$-ideal of $\mu=\mu(x, \cdot)$ null sets for $x \in X=f(Y)$. Using Lemma 2.1, we get (1) of Theorem 3.3 for all $1 \leq \alpha=\alpha^{*}<\omega_{1}$ again. Each 
$B_{x}$ is of positive measure $\mu=\mu(x, \cdot)$ for $x \in X=f(Y)$ by (b). Thus (2) of Theorem 3.3 is also satisfied, and we may use its conclusion also in this case with $\alpha+1$ instead of $\alpha$.

In both cases, due to Theorem 3.3, there is a selection $\xi$ for $f^{-1}(x)=B_{x}$ which is $\boldsymbol{\Sigma}_{\alpha+1}^{0}(X)$-measurable. The mapping $s: y \mapsto \xi(f(y))$ is $\boldsymbol{\Sigma}_{\alpha+\alpha+1^{-}}^{0}$ measurable by [5, §31, III, Theorem 2].

The point $s(y)$ is an element of the set $P \in \mathcal{P}$ which contains $y$ by the above Claim. Thus $s$ is a selector for the partition and the set $S=$ $\{y \in Y: y=s(y)\}$ is a selector set for the partition $\mathcal{P}$ which is of class $\Pi_{\alpha+\alpha+1}^{0}$ in $Y$. Indeed, the mapping $(y, z) \in Y \times Y \mapsto(s(y), z) \in Y \times Y$ is $\Sigma_{\alpha+\alpha+1}^{0}(Y \times Y)$-measurable (it suffices to check this on the preimages of sets of the form $\left.W_{n} \times W_{n^{\prime}}\right)$ and $S$ is the preimage of the closed diagonal $\{(y, y): y \in Y\}$ under it.

In the case $\alpha=2$ and for partitions into $G_{\delta}$ sets, a theorem by Miller [9. Theorem 1] gives a finer result, namely the existence of a $\boldsymbol{\Sigma}_{2}^{0}$-measurable selector $s: Y \rightarrow Y$ of the partition, i.e., a mapping such that $s(P)$ is a singleton in $P$. This indicates that our last corollary might not give the optimal estimates on the classes of selector mappings and selector sets even for higher classes $\alpha$.

Acknowledgments. I thank Piotr Borodulin-Nadzieja for the interesting question and Roman Pol for his hospitality and stimulating remarks during the preparation of this note during my stay in Warsaw in June 2008.

The work is a part of the research project MSM 0021620839 financed by MSMT and partly supported by GAČR 201/06/0198 and GAČR 201/06/ 0018.

\section{References}

[1] D. Blackwell and C. Ryll-Nardzewski, Non-existence of everywhere proper conditional distributions, Ann. Math. Statist. 34 (1963), 223-225.

[2] R. J. Gardner and W. F. Pfeffer, Borel measures, in: Handbook of Set-Theoretic Topology, North-Holland, Amsterdam, 1984, Chapter 22, 961-1043.

[3] P. Holický, Borel sets with countable sections for nonseparable spaces, Proc. Amer. Math. Soc. 134 (2006), 1519-1525.

[4] A. S. Kechris, Classical Descriptive Set Theory, Springer, New York, 1995.

[5] K. Kuratowski, Topology, Vol. I, Academic Press, New York, 1966.

[6] K. Kuratowski and C. Ryll-Nardzewski, A general theorem on selectors, Bull. Acad. Polon. Sci. 13 (1965), 397-403.

[7] W. Marciszewski and J. Pelant, Absolute Borel sets and function spaces, Trans. Amer. Math. Soc. 349 (1997), 3585-3596.

[8] R. D. Mauldin, Borel parametrizations, ibid. 250 (1979), 223-234.

[9] D. E. Miller, A selector for equivalence relations with $G_{\delta}$ orbits, Proc. Amer. Math. Soc. 72 (1978), 365-369. 
[10] H. Sarbadhikari, Some uniformization results, Fund. Math. 97 (1977), 209-214.

[11] S. M. Srivastava, A Course on Borel Sets, Springer, New York, 1998.

[12] - Selection theorems for $G_{\delta}$-valued multifunctions, Trans. Amer. Math. Soc. 254 (1979), 283-293.

Petr Holický

Department of Mathematical Analysis

Faculty of Mathematics and Physics

Charles University

Sokolovská 83

18675 Praha 8, Czech Republic

E-mail: holicky@karlin.mff.cuni.cz

Received 1 December 2008;

in revised form 1 September 2009 